\title{
DARPA und SF
}

\section{Erhard Taverna}

Dr. med., Mitglied der Redaktion

Ein Akronym für Defence Advanced Research Projects Agency. DARPA ist eine Institution des US-Verteidigungsministeriums. SF steht für Science Fiction. Geld ist kein Problem. DARPA investiert in Hunderten von Programmen, oft in Form eines Projektwettbewerbs, an dem sehr viele Universitäten teilnehmen. Elektronik, Nanotechnologie und Biotechnologie sind nur einige Beispiele für Forschungsthemen, die der nationalen Sicherheit dienen. Vom Stealth-Bomber über Wasseraufbereitung aus der Luft, vom Wiederherstellen geschredderter Dokumente bis zu biometrischen Erkennungssystemen, es gibt kaum einen Bereich, der nicht interessiert. Medizinische Projekte sind weit oben auf der Prioritätenliste, denn es geht um Restore and Maintain Warfighter Abilities. Dazu gehören vollautomatische Evakuierungssysteme mit Ganzkörperscans, die noch auf dem Transport telechirurgische Notfalleingriffe, nach dem Da-Vinci-Roboter-System, ermöglichen. Neuartige Prothesen sind im Test, bei denen die Sensibilität, vermittelt durch Handpropriozeption und Berührungspunkte, über im Hirn implantierte Module, die motorischen Funktionen verbessern. Trümmerfrakturen sollen, mit Hilfe völlig neuer Biomaterialien, in viel kürzerer Zeit verheilen, programmierbare Mikroben Infektionen bekämpfen. Seit Präsident Obama im April 2013 eine Brain Initiative ankündete, sind viele Technologiefirmen, akademische Institutionen und Neurowissenschaftler auf der Suche nach neuartigen Anwendungen. Vieles, was DARPA entwickelt, könnte man als Mensch-Maschinen-Symbiosen bezeichnen. Zum Beispiel neurologische Implantate im Hippocampus, um den Gedächtnisverlust nach einem Hirntrauma, Alzheimer oder Epilepsie zu beheben. Im Programm ist eine Miniatursonde, die drahtlos eine telemetrische Stimulation der betroffenen Areale ermöglicht, um alte Erinnerungsinhalte wieder herzustellen und neue zu ermöglichen. Die Projektmacher haben keine Berührungsscheu mit Science-Fiction-Literatur. Im Gegenteil, sie wird systematisch nach Ideen abgesucht. Technovelgy, tech-novel-gee, soll kreative, futuristische Vorhaben stimulieren und wohl auch interessierte Sponsoren, über deren Lieblingslektüre, in die Projekte einbinden. Star Wars, Kampfdroiden und Klonkrieger weisen den Weg zur Lösung von Problemen mit Drohnen-Armeen und intelligenten Robotern. Was DARPA an Sensoren entwickelt, fördert den zivilen
Gebrauch und umgekehrt. iPhones werden über Apps zu Messgeräten. Mit Quantified Self Movement entsteht ein riesiges Angebot für Selbstvermesser, die ihre Daten online zur Verfügung stellen. Die Technologiegläubigkeit macht keinen Unterschied zwischen militärischer und ziviler Anwendung. Dual Use macht es unmöglich, und faktisch oder zumindest mental ist die Mensch-Maschinen-Symbiose alltäglich. Sie wird zur Selbstverständlichkeit, denn ohne sie scheint keine zivilisierte Zukunft denkbar. Für Sicherheit und Gesundheit, individuell oder kollektiv, ist jede Kontrolle gut. Der Cisco Visual Networking Index liefert jährliche Trendanalysen. Bis in einem Jahr nutzen dreimal so viele Geräte einen Internetanschluss, wie Menschen auf der Erde leben. Der zukünftige Haushalt misst sich an seinem monatlichen Gigabytes-Umsatz. Diese selbstgefertigte Kunstwelt umgibt uns wie ein undurchdringlicher Kokon. Wir alle erfahren die Welt aus zweiter und dritter Hand.

Nur vor dieser Entwicklung ist der Erfolg eines Bestsellers wie The Martian von Andy Weir erklärbar. Eine Robinsonade auf dem Mars, die ausschliesslich technisch beschrieben wird. Der Protagonist, Botaniker und Mechaniker, werkelt wie Daniel Düsentrieb, stellt Treibstoff und Wasser her, pflanzt Kartoffeln und errechnet kaloriengenau seine Überlebenschancen. Eine Parallelhandlung auf der Erde ergänzt den Überlebenskampf mit einem perfekten NASA-Team, unzähligen Spezialisten und global wirksamer Medieninformation. Robinson geht mit Emotionen sehr sparsam um. Der Held gleicht mehr einem Roboter, der mit immer neuen Komplikationen fertig werden muss. Ein technisches Planspiel in einer lebensfeindlichen Umwelt. Sinn und Zweck des milliardenteuren Unternehmens werden nie reflektiert, ein Astronaut ist auch Soldat. Wer, wie in diesem Abenteuer, punktgenau Acidalia Planitia auf dem Mars ansteuert, kann auch zielgenau jeden Erdenwinkel ins Visier nehmen. Hier treffen sich nahtlos DARPA und SF. Nichts illustriert das besser als dieser mehrfach übersetzte und neuerdings auch verfilmte Roman. Der Schwerpunk liegt auf Science der Jetztzeit. Die Fiction hat einen Zeithorizont von zwanzig bis dreissig Jahren. Vielleicht können Ingenieure vom Informatiker-Autor noch etwas dazulernen. Leser, es sind bestimmt mehr Männer als Frauen, die das Buch oder den Film konsumieren, können grenzenlos ihr Technikvisionen ausleben. 